Publications of the Astronomical Society of the Pacific, 119: 886-897, 2007 August

(C) 2007. The Astronomical Society of the Pacific. All rights reserved. Printed in U.S.A.

\title{
The Tennessee State University Automatic Spectroscopic Telescope: Data Processing and Velocity Variation of Cool Giants
}

\author{
Joel A. Eaton and Michael H. Williamson \\ Center of Excellence in Information Systems, Tennessee State University, Nashville, TN; eaton@donne.tsuniv.edu \\ Received 2007 April 26; accepted 2007 June 28; published 2007 August 29
}

\begin{abstract}
This paper discusses data reduction for an echelle spectrograph we have developed for an automatic telescope at Tennessee State University and are using to monitor radial velocities and line profiles of cool giant and supergiant stars. Although our approach to data reduction is rather conventional, we discuss flatfielding and extraction of velocities in ways that should be of general interest, establish a transformation to the IAU radial velocity system $\left(+0.35 \pm 0.09 \mathrm{~km} \mathrm{~s}^{-1}\right)$, and determine the external precision for measured velocities $\left(0.10-0.11 \mathrm{~km} \mathrm{~s}^{-1}\right)$. Also, we present results of the first 2-3 years of monitoring radial velocities in about 120 cool giants and compare those results with the level of variability found with photometry. These new data confirm the widely held understanding that $\mathrm{K}$ and $\mathrm{M}$ giants are all radial velocity variables at the level of $0.1 \mathrm{~km} \mathrm{~s}^{-1}$.
\end{abstract}

\section{INTRODUCTION}

At the Center of Excellence in Information Systems at Tennessee State University, we have been operating robotic telescopes for the past 18 years, mostly moderately sized ones for aperture photometry (Henry 1995; Eaton et al. 2003). As part of our commitment to building and maintaining a completely automatic observatory with which we can study astronomical phenomena simultaneously with imaging, spectroscopy, and photometry, we have added a $2 \mathrm{~m}$ telescope for high-dispersion spectroscopy. All these instruments operate autonomously under computer control at Fairborn Observatory, a private site in southern Arizona.

This paper gives details of the data handling and reduction for spectra from our $2 \mathrm{~m}$ telescope. For information about how we control and manage this telescope, you may refer to Eaton $\&$ Williamson (2004a, 2004b). We discuss the reduction of the spectra in $\S 2$, calibration of the spectra in $\S 3$, and measurement of radial velocities for cool stars in $\S 4$, including expected external precision and systematic errors. Section 5 gives some results from measuring velocities of cool giant and supergiant stars.

The $2 \mathrm{~m}$ Automatic Spectroscopic Telescope (AST; Fig. 1) is designed specifically for automation of its primary task, obtaining spectra. It is a classical Cassegrain with an $\mathrm{f} / 1.5$ primary mirror and an aluminum secondary, giving an overall $\mathrm{f} / 8$ optical system. The focal plane is in front of the primary mirror, which reduces the size and weight of the secondary, making it cheaper and easier to support and, incidentally, reduces the pressure to lard other instruments onto the telescope. We support the secondary with a quadrupod structure, which reduces physical interference between the telescope tube and the enclosure. As a result, we are able to house the telescope in a relatively small building with a roll-off upper section. The AST acquires stars with a small acquisition/guiding camera, looking at the telescope focus through a $45^{\circ}$ pick-off mirror and transfer optics, and sends light to the spectrograph through a $200 \mu \mathrm{m}$ fiberoptic cable projecting through a hole in this mirror. The telescope guides on light spilling over the edge of the fiber (Libbrecht \& Peri 1995). For wavelength calibration/flat-fielding, we focus light at $\mathrm{f} / 8$ onto the fiber feeding the spectrograph, which we bring into the guiding head from bench-mounted sources through a $600 \mu \mathrm{m}$ fiber.

The spectrograph itself (Fig. 2) is a cross-dispersed echelle of rather conventional white-pupil design (Dekker et al. 1992; Pilachowski et al. 1995) based on commercially available gratings. The camera is a custom design by H. Epps, similar to the camera he designed for the Hobby-Eberly Telescope (Epps 1998), but with some of the glasses changed to extend transmission into the near-ultraviolet. This spectrograph has two wavelength settings chosen by rotating the cross-dispersion grating, a red position with wavelengths $5000-7100 \AA$ (covering $\mathrm{H} \alpha, \mathrm{Na}$ I $\mathrm{D}, \mathrm{Li}$ I $\lambda 6708$, and various $\mathrm{TiO}$ bands) and a blue one for 3700-5700 $\AA$ (covering Ca II $H$ and $K$, the higher Balmer lines, and a few lines of singly ionized metals in the $\zeta$ Aur binaries). We normally operate it in a standard mode with a resolution of $R \approx 30,000$, based on feeding the spectrograph with a bare $200 \mu \mathrm{m}$ fiber. There is a second mode with somewhat higher resolution, based on feeding the spectrograph with a $75 \mu \mathrm{m}$ slit in front of a second fiber. We record the spectra with a large-format CCD. The initial program for the AST consists primarily of measurements of radial velocities of long-period spectroscopic binaries and monitoring of $\mathrm{H} \alpha$ in cool stars.

For the most part, the AST is a very conservative design. 


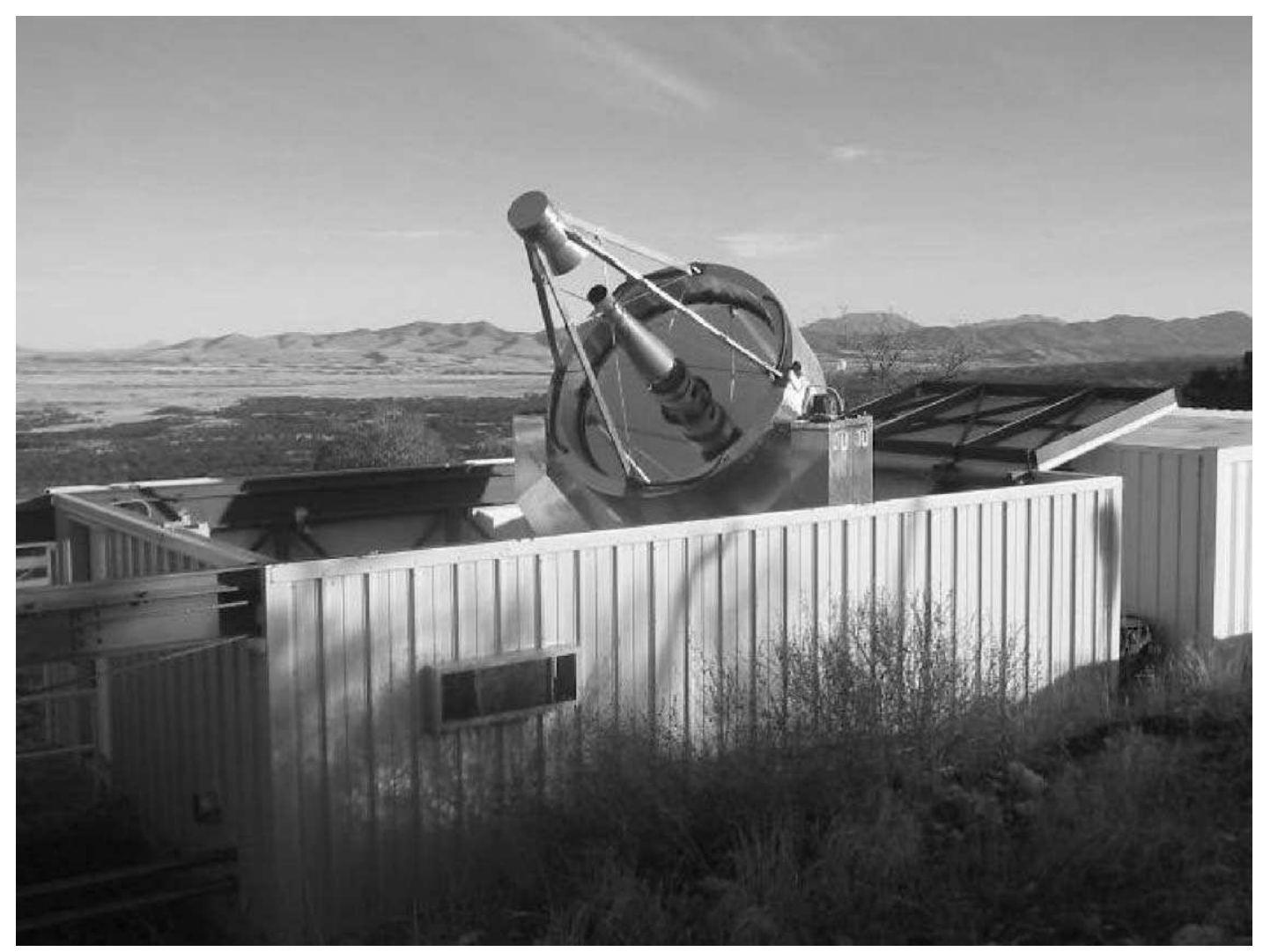

FIG. 1.-TSU 2 m AST at Fairborn Observatory. The telescope is housed in a barn-like enclosure with a top section that rolls off to the north. The echelle spectrograph is in an insulated shipping container to the south.

Possibly innovative features are (1) the quadrupod mount for the secondary mirror, which L. J. Boyd had previously used in photometric telescopes, (2) having the focal plane between the two mirrors to make the secondary mirror smaller and the telescope tube lighter, and (3) the use of an enclosure too small to shut unless the telescope is properly stowed. We think this is the first completely automatic telescope to be implemented with an altitude-azimuth mount. The only signifi cant technical risk was our scheme for guiding on light slopping over the edge of the fiber, but Libbrecht \& Peri had shown this technique can work, and we had amazingly little trouble implementing it.

\section{DATA REDUCTION}

We use a data reduction program based on the one written at Lowell Observatory by J. Hall for the Solar-Stellar Spectrograph (Hall et al. 1994; Hall \& Lockwood 1995). It takes the usual steps in this process: (1) bias subtraction, (2) mapping the orders, (3) subtracting the background of scattered light, (4) fht-fi elding, (5) collapsing the image into a two-dimensional array, (6) wavelength calibration, and (7) archiving. Hall originally wrote this program in IDL (Interactive Data Language from Research Systems, Inc.), but we rewrote it in C to rid our observatory of proprietary software with its licensing problems.

At both the beginning and end of each night, we take 8 bias frames (readout of CCD without exposure), 16 fht-fi eld frames (exposed to a continuous source as described below), and one wavelength calibration frame (thorium-argon hollow cathode lamp). These are then reduced to an average bias frame for the night (average of 16 images) and average fhts for the beginning and end of the night (4 images averaging 8 frames each). Such averaging lets the reduction program fi lter out cosmic-ray hits and allow for bad pixels.

Figures 3 and 4 show some properties of the fht-fi eld spectra. Figure 3 is a cut through the orders in two averaged fht-fi eld frames for Modifi ed Julian Date 53,925 to show the separation of the orders. The curve with the very broad orders is the one actually used for fht-fi elding. Superimposed is a traditional fht fi eld used to defi ne the location of the orders. The top panel of Figure 4 gives the run of intensity versus position in the fht-fi eld spectrum for several orders to show the complicated variation of intensity with wavelength, the echelle blaze function.

In the fi rst step, the reduction program subtracts the average bias frame, pixel by pixel, from the average fhts, the wave- 


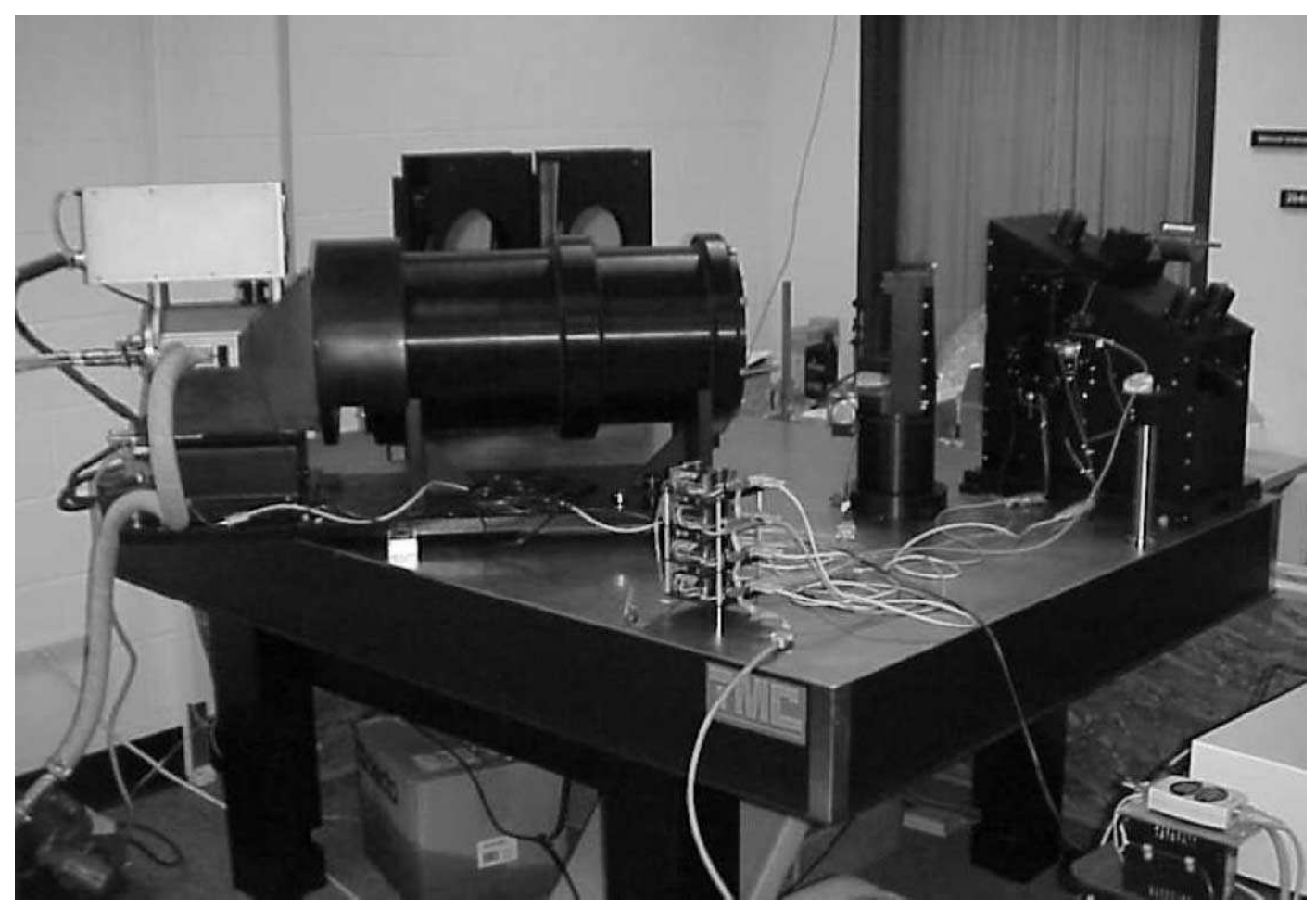

FIG. 2.-Echelle spectrograph for the TSU $2 \mathrm{~m}$ automatic telescope assembled in the lab at TSU.

length calibration frames, and the individual frames for the stars. After this debiasing, scattered light is removed from each star's spectrum by subtracting the value of a pixel in between the orders. Defi ning the scattered light in this spectrograph is made much easier by the relatively large separation of the orders (Fig. 3). We use an offset of \pm 13 pixels in the direction

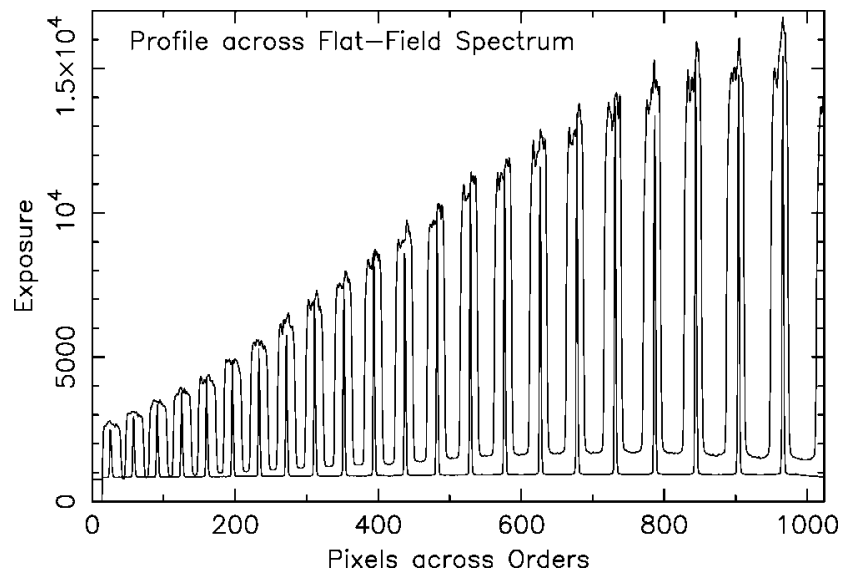

FIG. 3.-Profi le of the echelle spectrum across the dispersion. The broad profi les are for the image of an incandescent source with the camera out of focus, a flat-fi eld image. The sharp profi les are for a focused image of this source, which we use for locating the orders and calibrating the echelle blaze function. perpendicular to the echelle orders, which also helps to remove the camera's periodic readout noise.

For the flat-fi elding, we take two types of images each night, as illustrated in Figure 3. For the fi rst kind, light from an incandescent bulb is passed through the spectrograph, with the camera focused normally. These images are used to locate the echelle orders and to normalize the extracted spectra. The original version of the program also used them as fht fi elds for correcting pixel-to-pixel sensitivity variations. However, in that original version, this correction was applied only after the orders had been collapsed to a single value by summing across each order perpendicular to the echelle dispersion axis. The averaged fht fi eld was collapsed in this way, and a piecewise continuous spline function was fi t to each order to estimate an actual intensity curve. From this, a correction could be calculated and applied to the extracted spectra of the stars. That method depended on there being no drift in position between the fht-fi eld spectra and the stars' spectra during the night, although that is not usually the case. Also, we have since found that these flat fi elds contain periodic noise at the level of about $1 \%$, with a period of $\sim 1$ cycle $\AA^{-1}$.

In the current version of the program, we use a second type of fht fi eld, taken by putting the camera out of focus. We chose our out-of-focus position to illuminate all the pixels used to measure the stellar spectra suffi ciently well to defi ne the individual pixel sensitivity reliably for them. For these fi elds, the 

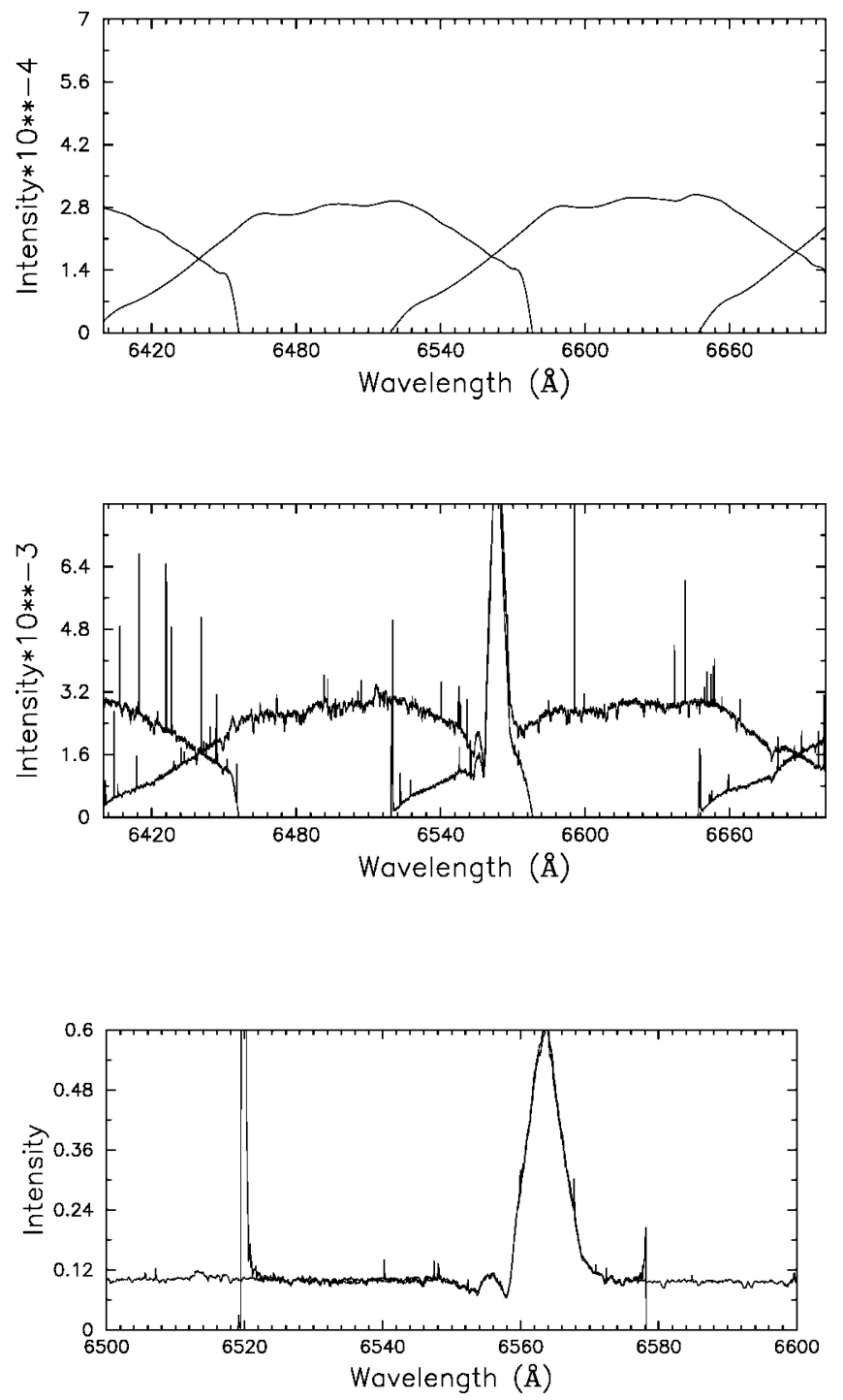

FIG. 4.-Effect of the echelle blaze function on the spectrum. In the top panel, we see the spectrum of the incandescent fht-fi eld lamp (orders 54-51) showing the variation in response with wavelength. In the lower panels, we have an observation of the interacting binary AX Mon. The middle panel is the star's spectrum cleared of scattered light, while the lower panel shows that spectrum divided by the reduced fat to remove most of the variation of sensitivity with wavelength.

actual intensity received by a pixel is estimated by taking the average of the median third of the adjacent 100 pixels in the same column. The CCD is rotated slightly in the camera so that the echelle orders are nearly parallel to the CCD columns, so a column of 100 pixels has somewhat uniform illumination. Pixel sensitivity corrections are calculated this way for 16 outof-focus fats, and the median of these is taken and used to correct all of the star spectra for the night. Gradual changes in pixel sensitivity, caused partly by material condensing and evaporating from the surface of the CCD, occur rapidly enough that we must take new flat fi elds every night.
Once the intensity in the spectra is corrected by flat-fi elding, the program collapses each image into a one-dimensional array consisting of wavelength-calibrated spectra of the various orders. The middle panel in Figure 4 gives an example of these. The fi nal step in the basic reduction is to correct for the rather ugly variations of sensitivity along the orders by normalizing to the average focused fht-fi eld image for the whole night, reduced in the same way as the stellar spectra. An example of such an image is shown in the top panel of Figure 4, and the result of the normalization in the bottom panel.

For wavelength calibration, we use 10 to 15 relatively bright and unblended ThAr lines for each echelle order. The program determines the pixel center of each ThAr line in the direction of dispersion with a Gaussian fi t. It then fi ts the known wavelengths of these lines, taken from a ThAr atlas, with a fourthorder polynomial. This is normally done for two ThAr spectra each night, one at the beginning and one at the end of the night.

We do all of the aforementioned steps in the reduction automatically at the end of the night at the observatory, then copy two forms of reduced spectra back to TSU for archiving and analysis. These are (1) the wavelength-calibrated spectra unnormalized for intensity variation along the orders and (2) the fi nal wavelength-calibrated normalized spectra. We store these as FITS fi les consisting of two arrays, intensity versus pixel and wavelength versus pixel. We have purposely avoided mapping the spectrum onto a grid of uniformly spaced wavelengths at this stage because the wavelength calibration of any order is not linear and because the spacing is roughly proportional to wavelength. Also, directly recording the wavelengths eliminates questions about the wavelength scale in the future.

Once we copy the reduced spectra and certain quality-control data to TSU over the Internet, we run programs that look for deterioration of the system, such as an increasing temperature of the CCD or a calibration lamp that has burned out, and knit the normalized reduced spectra together onto a single wavelength scale that averages overlapping parts of the orders and cuts out the unreliable data at the ends of the orders. These knitted spectra are the primary product we use in analyses of AST data.

\section{INTENSITY AND WAVELENGTH CALIBRATION}

We use two types of calibration spectra, an incandescent lamp for intensity calibration (fht-fi elding) and a ThAr lamp for wavelength calibration. To illuminate the spectrograph as consistently as possible, we carry the calibration light up to the guiding head in the spectrograph with a separate fi ber optic cable and project it into the fi ber feeding the spectrograph at $\mathrm{f} / 8$, the focal ratio of the telescope. This scheme seems the best one can do to fi rst order, but there will be obvious difference between the distribution of calibration light and starlight, even with this sort of illumination (Griffi $n$ \& Griffi $n$ 1973).

Flat-fi elding in spectroscopy is quite unsatisfying conceptually, as can be seen by contrasting it with "imaging." In taking 
normal images of the sky with a CCD, one simply gathers a bunch of flat fi elds for a particular wavelength at the beginning of an observing run and uses them to correct for small-scale variations in sensitivity that show up as noise in an image. Of course, there are problems such as getting the right illumination of the CCD and the effect of internal reftections in the telescope, but these can be calibrated by cleverly using multiple observations of star clusters (Boyle et al. 2003). Flat-fi elding in spectroscopy is much more diffi cult for two reasons. First, the wavelength is continually changing over the CCD. Second, one cannot simply fbod the CCD uniformly as in "imaging," but must in practice illuminate the CCD through the spectrograph. This impresses the same uneven exposure across the spectrum as one gets for a star and makes it impossible to calibrate variations in sensitivity directly as in imaging. Instead, reduction programs for spectroscopy have complicated routines to take out the effect of cross-order illumination, which necessarily introduce noise with respect to uniform illumination.

One might get around this problem of cross-order illumination by illuminating the CCD uniformly with a separate source in the spectrograph, if the small-scale variations in sensitivity were independent of wavelength. Unfortunately, common knowledge has it that these variations are rather dependent on wavelength. To test this notion, we fboded our CCD with light from four types of light-emitting diodes (LEDs), diffused off a screen in front of the cross-dispersion grating, and compared the images. The four colors all showed most of the same features, but with variations at the level of a few percent. So, although this approach would probably work well for a wavelength range of a few hundred $\AA$, the variation in sensitivity was clearly too great for us to use it to reduce echelle spectra.

Another approach to the conceptual problem of cross-order illumination is to broaden the calibration spectrum either with a cylindrical lens or by putting the camera out of focus. We have chosen to apply that approach by throwing the spectrum out of focus as explained above. This leaves something to be desired, in that we are obviously not getting a uniform illumination perpendicular to the dispersion, as shown in Figure 3, but it does give plenty of light of the right wavelength at every position in the spectrum, while it requires no new parts for the spectrograph. For these reasons, we have decided to use it in our data reduction.

\section{MEASURING RADIAL VELOCITIES}

The velocity scale for the spectra rests on two kinds of observations: (1) ThAr calibration lines measured at the beginning and end of the night, and (2) the telluric $\mathrm{O}_{2}$ lines available in each individual spectrum. The wavelengths, hence velocities, are dependent on ThAr lines to establish the wavelengths of various echelle orders with respect to one another and telluric lines to correct for drifts during the night from such sources as thermal changes in the CCD. In practice, we use 273 calibration lines for 20 orders of the echelle spectrum in the red and 329 lines for 32 orders in the blue. There are almost certainly systematic changes in the velocity zero point among the orders. However, if these lines are applied consistently, they would defi ne a stable wavelength scale for which we can determine a meaningful absolute zero-point correction.

To measure the actual velocities of stars, we use a group of 74 strong metallic lines in the spectrum of the Sun, mostly Fe I, which we represent as delta functions at the wavelengths given by Moore et al. (1966), and cross-correlate them with the observed spectrum as a function of velocity shift. We then fi t the resulting cross-correlation function (CCF) with a Gaussian to get a velocity. For telluric $\mathrm{O}_{2}$, we use 32 lines in the range 6870-6924 $\AA$, again calculating a CCF and fi tting it with a Gaussian to get a velocity shift. Using this set of strong lines defi nes an average line profi le for the star, eliminates noise from weak lines, and means we can apply a common technique for a wide range of spectral type.

Using telluric lines to defi ne the velocity scale seems to be a natural thing to do, and indeed several authors (Hatzes \& Cochran 1993; Kamper \& Fernie 1998, for example) have actually done so, as discussed by Gray \& Brown (2006). Griffi n \& Griffi n (1973) comprehensively discussed this approach early on, fi nding that the winds in Earth's atmosphere can give systematic effects of up to $\sim 0.03 \mathrm{~km} \mathrm{~s}^{-1}$. Observations of the Sun confi rm that expectation (Balthasar et al. 1982; Kobanov 1985; Deming et al. 1987). This dependency limits the precision attained without actually measuring the atmospheric wind profi le, but the external precision of the velocities we have derived without such corrections is still several times as good as we could get from the ThAr calibration alone. Furthermore, it has the operational/maintenance advantage of obviating the need to take many calibration spectra during the night.

\subsection{Transformation to the IAU Velocity Scale}

The real challenge of calibrating the radial velocity system is defining an absolute zero point for heliocentric velocities that can be used to compare one's own velocities with others'. Heliocentric radial velocity is an absolute quantity, unlike the magnitudes of the $U B V$ photometric system (Johnson \& Morgan 1953). Complicating the defi nition of a zero point is the problem that knowledge about standardization generally appears through IAU meetings. Nevertheless, there is a de facto IAU velocity system based on a group of standard stars, somewhat like $U B V$ standards, which one can use to transform radial velocities for a particular instrument to a standard scale. These stars are given by Pearce (1957) and Bouigue (1973), and there are extant reports of the unsuitability of various ones as standards because of "excess" variability (e.g., Batten et al. 1983). Scarfe et al. (1990) give a rather intelligent comprehensive discussion of these standards as a group, comparing the canonical velocities with new measurements from Dominion Astrophysical Observatory (DAO). Stefanek et al. (1999) have published similar observations from Harvard. The existing 
TABLE 1

ObServations of Velocity "Standards"

\begin{tabular}{|c|c|c|c|c|c|c|}
\hline $\begin{array}{l}\text { HD } \\
(1)\end{array}$ & $\begin{array}{l}\text { Name } \\
(2)\end{array}$ & $\begin{array}{l}\text { Spectrum } \\
\text { (3) }\end{array}$ & $\begin{array}{l}N \\
(4)\end{array}$ & $\begin{array}{c}\mathrm{RV}_{\mathrm{AST}^{\mathrm{a}}} \\
\left(\mathrm{km} \mathrm{s}^{-1}\right) \\
(5)\end{array}$ & $\begin{array}{c}\text { AST - IAU } \\
\left(\mathrm{km} \mathrm{s}^{-1}\right) \\
(6)\end{array}$ & $\begin{array}{c}\text { AST - DAO } \\
\left(\mathrm{km} \mathrm{s}^{-1}\right) \\
(7)\end{array}$ \\
\hline 4128 & $\beta$ Cet & K0 III & 22 & $12.87 \pm 0.09$ & -0.23 & -0.29 \\
\hline 12929 & $\alpha$ Ari & K2 III & 54 & $-14.84 \pm 0.18$ & -0.54 & -0.33 \\
\hline $18884 \ldots \ldots$ & $\alpha$ Cet & M1.5 IIIa & 119 & $-26.52 \pm 0.27$ & -0.72 & -1.22 \\
\hline 29139 & $\alpha$ Tau & K5 III & 115 & $54.14 \pm 0.22$ & 0.04 & -0.11 \\
\hline 62509 & $\beta \mathrm{Gem}$ & K0 IIIb & 141 & $3.07 \pm 0.11$ & -0.23 & -0.16 \\
\hline 81797 & $\alpha$ Hyа & K3 II-III & 135 & $-4.54 \pm 0.18$ & -0.14 & -0.29 \\
\hline 84441 & $\epsilon$ Leo & G1 II & 127 & $4.04 \pm 0.15$ & -0.76 & -0.36 \\
\hline $102870 \ldots \ldots$ & $\beta$ Vir & F8 V & 65 & $3.83 \pm 0.11$ & -1.17 & -0.55 \\
\hline $124897 \ldots \ldots$ & $\alpha$ Boo & K1 III & 153 & $-5.55 \pm 0.21$ & -0.25 & -0.25 \\
\hline $146051 \ldots \ldots$ & $\delta \mathrm{Oph}$ & M0.5 III & 128 & $-19.80 \pm 0.24$ & 0.00 & -0.66 \\
\hline $161096 \ldots \ldots$ & $\beta \mathrm{Oph}$ & K2 III & 17 & $-12.59 \pm 0.09$ & -0.59 & -0.41 \\
\hline $186791 \ldots \ldots$ & $\gamma \mathrm{Aql}$ & K3 II & 119 & $-3.03 \pm 0.47$ & -0.93 & -1.06 \\
\hline $204867 \ldots \ldots$ & $\beta$ Aqr & G0 Ib & 121 & $5.98 \pm 0.23$ & -0.72 & -0.73 \\
\hline $222368 \ldots \ldots$ & $\iota$ Psc & F7 V & 58 & $5.00 \pm 0.18$ & -0.30 & -0.60 \\
\hline $22484 \ldots \ldots$ & $10 \mathrm{Tau}$ & F9 V & 63 & $27.52 \pm 0.10$ & -0.38 & -0.43 \\
\hline $26162 \ldots \ldots$ & 43 Tau & K2 III & 30 & $24.34 \pm 0.38$ & 0.44 & -0.45 \\
\hline $66141 \ldots \ldots$ & HR 3145 & K2 III & 53 & $71.40 \pm 0.15$ & 0.50 & -0.05 \\
\hline $103095 \ldots \ldots$ & HR 4550 & G8 Vp & 14 & $-98.81 \pm 0.23$ & 0.29 & -0.23 \\
\hline $107328 \ldots \ldots$ & $16 \mathrm{Vir}$ & K1 III & 49 & $36.18 \pm 0.23$ & 0.48 & -0.30 \\
\hline $115521 \ldots \ldots$ & $\sigma \mathrm{Vir}$ & M2 IIIa & 122 & $-27.58 \pm 0.60$ & -0.78 & -0.30 \\
\hline $136202 \ldots \ldots$ & $5 \mathrm{Ser}$ & F8 III-IV & 50 & $53.87 \pm 0.15$ & 0.37 & -0.58 \\
\hline $187691 \ldots \ldots$ & $o$ Aql & F8 V & 75 & $-0.53 \pm 0.13$ & -0.63 & -0.48 \\
\hline $212943 \ldots \ldots$ & $35 \mathrm{Peg}$ & K0 III & 54 & $54.00 \pm 0.14$ & -0.30 & -0.26 \\
\hline
\end{tabular}

${ }^{a}$ Raw velocity; not on IAU system.

"standard" velocities for these stars have not yet been placed on an absolute scale by tying them in to precise, dynamically determined velocities of objects in the solar system, for example, (cf. Scarfe 1985) so they cannot be any better in an absolute sense than well-determined velocities from other carefully calibrated spectrographs, such as the DAO spectrograph or our own. However, as a group, they do defi ne a system with a common, if unknown, zero point. Many of the IAU standards are variable at the level of a few tenths of $\mathrm{km} \mathrm{s}^{-1}$, and the discussion of Scarfe et al. makes clear that there must be random errors in the zero point within the group at this level. So, observations of one or a few individual IAU standards would not be suffi cient for establishing the zero point for a spectrograph or, for that matter, for an individual observing run. However, averaging observations of many of them should give a transformation to this IAU "system" valid at the level of roughly $0.1 \mathrm{~km} \mathrm{~s}^{-1}$.

We have observed the 23 IAU standards listed in Table 1 in

TABLE 2

Average Deviations from Standards

\begin{tabular}{lcc}
\hline \hline & $\begin{array}{c}\text { AST - IAU } \\
\left(\mathrm{km} \mathrm{s}^{-1}\right)\end{array}$ & $\begin{array}{c}\text { AST - DAO } \\
\left(\mathrm{km} \mathrm{s}^{-1}\right)\end{array}$ \\
\hline Weighted $\ldots \ldots \ldots \ldots \ldots$ & $-0.35 \pm 0.42$ & $-0.36 \pm 0.13$ \\
Unweighted $\ldots \ldots \ldots \ldots$ & $-0.28 \pm 0.48$ & $-0.44 \pm 0.28$ \\
Stars in common $\ldots \ldots . .$. & 23 & 23 \\
\hline
\end{tabular}

Note.-Quoted uncertainties are the standard deviation of a single star from the average. various programs over the fi rst 2 years of collecting data with the telescope, primarily as part of our synoptic program and as comparison stars for spectroscopic binaries we were observing for F. C. Fekel. This is essentially a reprise of Scarfe et al. (1990). The values in column (5) are all averages of the $N$ observations, measured with the cross-correlation analysis described three paragraphs ago; quoted uncertainties are the standard deviation of a single measurement from the mean. (We did this analysis some time before our analysis of variability in Table 3, and with different criteria for including data; hence, the smaller number of stars included here.) We have also listed differences between our values and the canonical IAU velocities in column (6) and with respect to Scarfe et al. in column (7). We may use the averages of these two columns in Table 2 to estimate the zero-point correction to the IAU system and get some idea of its reliability. The fi rst value for these differences is weighted as $\sigma^{-2}$, where $\sigma$ is the value for a single measurement to refect the likely intrinsic variability of the star. The uncertainties quoted for these averages are again standard deviations for a single star. The average values should be about 5 times as precise. We may note that the DAO velocities are likely much better than the IAU values, but both groups give about the same transformation to the IAU system. We conclude that the AST gives velocities $0.35 \pm 0.09 \mathrm{~km}$ $\mathrm{s}^{-1}$ more negative than the canonical IAU values, so we would add $0.35 \mathrm{~km} \mathrm{~s}^{-1}$ to our measured values to bring them onto the IAU system. This measured difference is close to the de- 
viation that Scarfe (1985) found between the IAU system and some estimates of absolute velocities, $+0.35 \pm 0.18 \mathrm{~km} \mathrm{~s}^{-1}$.

\subsection{External Errors of Velocities}

We have estimated the external random errors of our velocities as measured by the system of solar photospheric lines and telluric $\mathrm{O}_{2}$ lines in two ways. First, we looked at the standard deviations about their means for the stars in our sample with the most constant velocities. Such stars have standard deviations near $0.1 \mathrm{~km} \mathrm{~s}^{-1}$. Examples from Table 3, which gives results of our primary monitoring program, are $\alpha$ Ari (K2 IIIab; $\sigma=0.11$ ), $\beta$ Gem (K0 III; $\sigma=0.11$ ), $\gamma$ Psc (K0 III; $\sigma=$ 0.13 ), HD 6833 (G9 III; $\sigma=0.14$ ), $\kappa$ Aur (G8 III; $\sigma=0.14$ ), $\zeta$ Hya (G9 II-III; $\sigma=0.14$ ), $\epsilon$ Leo (G1 II; $\sigma=0.14$ ), $\epsilon$ Boo (K0 II-III; $\sigma=0.14$ ), $\beta$ Boo (G8 III; $\sigma=0.14$ ), and $\delta$ Cnc (K0 III; $\sigma=0.15$ ). Others from Table 1 , typically with fewer observations, are $\beta$ Cet (K0 III; $\sigma=0.09$ ), $\beta$ Vir (F8 V; $\sigma=$ 0.11 ), $\beta$ Oph (K2 III; $\sigma=0.09$ ), 10 Tau (F9 V; $\sigma=0.10$ ), and $o$ Aql (F8 V; $\sigma=0.13)$. Even among the quietest of these, $\alpha$ Ari and $\beta$ Gem are known to vary at the $0.05 \mathrm{~km} \mathrm{~s}^{-1}$ level (Walker et al. 1989), and at least some of the variation in all of these nearly constant stars must be intrinsic. We can get a further idea about the external errors by measuring deviations of velocities of spectroscopic binaries from fi tted velocity curves. Two examples of this are our data for 52 Per (G5 $\mathrm{Ib}+\mathrm{A} 2 ; \sigma=0.117)$ and $\eta$ Peg $(\mathrm{G} 2 \mathrm{II}+\mathrm{F} ; \sigma=0.141)$. In addition, we have analyzed 169 measurements of spectra we took for F. C. Fekel (see Fekel et al. 2007 for an offi cial orbit) for HD 14214 (G0.5 IV), for which the standard deviation from the solution is $\sigma=0.095 \mathrm{~km} \mathrm{~s}^{-1}$. Figure 5 shows the deviations of that fit as a function of time. There is little, if any, phase dependence. There may be a seasonal effect in the second full year of data, but it is at the $0.05 \mathrm{~km} \mathrm{~s}^{-1}$ level. Such small $\sigma$ 's are found only for sharp-lined stars. This could be because our technique does not work as well for broad-lined stars (supergiants, moderately rapid rotators), or it could be that these broader-lined stars are actually variable at the $0.2 \mathrm{~km} \mathrm{~s}^{-1}$ level. We may discriminate between these two possibilities by looking for warmer broad-lined supergiants not expected to have irregular pulsations, such as Cepheid variables. As our one good example, Polaris (F7 Ib-II) gives velocities for data divided into 8 sets of $\sim 55$ measurements each, for which deviations from a fit thed sine curve give $\sigma$ in the range $0.083-0.165 \mathrm{~km}$ $\mathrm{s}^{-1}$, with a median of $0.111 \mathrm{~km} \mathrm{~s}^{-1}$. The average velocities for these sine curves follow the motion in a well-known longperiod orbit (Kamper 1996) to $0.15 \mathrm{~km} \mathrm{~s}^{-1}$. Well-observed spectroscopic binaries give some further cases. One is $5 \mathrm{Cet}$, a binary with $v \sin i=22 \mathrm{~km} \mathrm{~s}^{-1}$, for which the data scatter by roughly $\pm 0.10 \mathrm{~km} \mathrm{~s}^{-1}$ (see $\S 5.1$ and Eaton 2007). The three classical $\zeta$ Aur binaries, $\zeta$ Aur, $31 \mathrm{Cyg}$, and $32 \mathrm{Cyg}$, show systematic deviations from their orbital motion of $\sim \pm 0.5 \mathrm{~km}$ $\mathrm{s}^{-1}$, with a scatter of $\sim \pm 0.1 \mathrm{~km} \mathrm{~s}^{-1}$ about the trend of those deviations (J. A. Eaton et al. 2007, in preparation).
The evidence discussed above argues that $\sigma_{\text {ext }}$ is tending asymptotically toward $0.10 \mathrm{~km} \mathrm{~s}^{-1}$ with decreasing intrinsic stellar velocity variation. On this basis, we conclude that the external error of an observed velocity is conservatively 0.10 $0.11 \mathrm{~km} \mathrm{~s}^{-1}$ for those stars for which the solar mask spectrum is appropriate, namely those with moderately sharp lines of spectral type $\mathrm{F}$ to middle $\mathrm{M}$.

\section{VARIABILITY OF COOL GIANTS}

We have observed a group of about $120 \mathrm{cool}$ giant stars over the first 2-3 years of telescope operation to study their orbits, to detect their intrinsic variability, and to monitor their $\mathrm{H} \alpha$ lines for outbursts in winds. These are mostly $\mathrm{K}$ giants and supergiants, although the group includes a number of $\mathrm{G}$ and $\mathrm{M}$ stars as well. Of these stars, 15 may be constant at the $0.1 \mathrm{~km} \mathrm{~s}^{-1}$ level. See a list of these in $\S 4.2$ two paragraphs ago. However, almost all the $\mathrm{K}$ and early $\mathrm{M}$ supergiants we have observed seem to be variable at the level of $0.1-0.3 \mathrm{~km} \mathrm{~s}^{-1}$, as might be expected from previous surveys of such stars.

Table 3 gives some results for this group; Table 4, further results for several binaries. The radial velocity in column (6) of Table 3 is an average, with the standard deviation of a single measurement quoted as the uncertainty. For spectroscopic binaries, the standard deviation is with respect to the fi tted velocity curve, so the average velocity would be the $\gamma$-velocity from Table 4. The later M stars and carbon stars have spectra dominated by molecular lines, so we have fi gured their standard deviations by correlating the spectra of each star with the first observed spectrum of the star itself.

Cool giant stars seem to be universally variable, presumably from low-amplitude pulsation or asymmetries in convective motions (e.g., Gray \& Toner 1985, 1986a, 1986b). Others are demonstrably variable from starspots (e.g., Hall 1976; Queloz et al. 2001). Henry et al. (2000) investigated the photometric variability of a large sample of $\mathrm{G}-\mathrm{K}-\mathrm{M}$ giants and supergiants with highly precise differential photometry, fi nding that $43 \%$ of their sample were variable $(\sigma \geq 0.0020 \mathrm{mag})$ and that all the stars to the cool side of the Linsky-Haisch coronal dividing line are pulsating variables. Percy et al. (2001) measured photometric variability of M-giant variables, which seemed to be pulsating preponderantly in one or more radial modes.

The photometric variations are accompanied by velocity variations, and these are well known for cool supergiants. Walker et al. (1989), for instance, discussed the velocity variations of fi ve K giants and supergiants, and Smith et al. (1989) followed velocity variations of three $\mathrm{M}$ supergiants. Velocity surveys of $\mathrm{K}$ and M stars by Larson et al. (1999) and Cummings et al. (1999), with external errors of $\sim 0.01$ and $0.05 \mathrm{~km} \mathrm{~s}^{-1}$, respectively, show that the normal $\mathrm{K}$ giants are all likely to be variable at the $0.03 \mathrm{~km} \mathrm{~s}^{-1}$ level. This evidence, along with our own observations, contradicts a recent optimistic prediction (Bizyaev \& Smith 2007) that $12 \%-20 \%$ of K giants should have stable radial velocities. 
TABLE 3

Measured Velocities for Cool Giants

\begin{tabular}{|c|c|c|c|c|c|c|}
\hline $\begin{array}{l}\mathrm{HD} \\
(1)\end{array}$ & $\begin{array}{l}\text { Name } \\
(2)\end{array}$ & $\begin{array}{c}V \\
(3)\end{array}$ & $\begin{array}{c}\text { Spectral Type } \\
\text { (4) }\end{array}$ & $\begin{array}{c}N \\
(5)\end{array}$ & $\begin{array}{c}\mathrm{RV}_{\mathrm{AST}}^{\mathrm{a}} \\
\left(\mathrm{km} \mathrm{s}^{-1}\right) \\
(6)\end{array}$ & $\begin{array}{l}\text { Notes } \\
(7)\end{array}$ \\
\hline 352 & $5 \mathrm{Cet}$ & 6.22 & K2 III & 126 & $\ldots \pm 0.39$ & SB1 \\
\hline $1522 \ldots \ldots \ldots$ & $\iota \mathrm{Cet}$ & 3.56 & K1.5 III & 96 & $18.80 \pm 0.16$ & \\
\hline$\ldots \ldots \ldots$ & $\zeta$ And & 4.06 & K1 IIe & 115 & $\ldots \pm 0.42$ & SB1 \\
\hline $4656 \ldots \ldots \ldots$ & $\delta$ Psc & 4.43 & K5 III & 103 & $32.86 \pm 0.22$ & \\
\hline $6833 \ldots \ldots \ldots$ & & 6.77 & G9 III & 15 & $-243.40 \pm 0.14$ & \\
\hline $6860 \ldots \ldots \ldots$ & $\beta$ And & 2.06 & M0 IIIa & 107 & $-0.05 \pm 0.23$ & \\
\hline $8890 \ldots \ldots \ldots$ & $\alpha \mathrm{UMi}$ & 2.02 & F7 Ib-II & 402 & Var. \pm 0.11 & Cepheid \\
\hline$\ldots \ldots \ldots$ & & 5.70 & $\mathrm{~K} 0 \mathrm{Ib}+\mathrm{B} 9 \mathrm{~V}$ & 27 & $-12.21 \pm 0.39$ & \\
\hline $9927 \ldots \ldots \ldots$ & 51 And & 3.57 & K3 III & 63 & $16.28 \pm 0.20$ & \\
\hline $12533 \ldots \ldots$. & $\gamma^{1}$ And & 2.26 & K3 IIb & 78 & $-11.38 \pm 0.20$ & \\
\hline $12642 \ldots \ldots \ldots$ & & 5.62 & K5 I: & 108 & $24.98 \pm 0.28$ & \\
\hline $12929 \ldots \ldots \ldots$ & $\alpha$ Ari & 2.00 & K2 IIIab & 152 & $-14.50 \pm 0.11$ & \\
\hline $16115 \ldots \ldots \ldots$ & & 8.15 & $\mathrm{C} 2,3$ & 39 & $\ldots \pm 0.51$ & Self; $P \sim 100$ days \\
\hline $17709 \quad \ldots \ldots \ldots$ & 17 Per & 4.53 & K7 III & 81 & $13.54 \pm 0.21$ & \\
\hline $18884 \ldots \ldots \ldots$ & $\alpha$ Cet & 2.53 & M1.5 IIIa & 124 & $-26.17 \pm 0.25$ & \\
\hline $19058 \ldots \ldots \ldots$ & $\rho$ Per & 3.39 & M4 II & 71 & $28.01 \pm 0.90$ & \\
\hline $19476 \ldots \ldots \ldots$ & $\kappa$ Per & 3.80 & K0 III & 57 & $27.63 \pm 0.52$ & SB? $(P>450$ days $)$ \\
\hline $21552 \ldots \ldots \ldots$ & $\sigma$ Per & 4.36 & K3 III & 52 & $14.38 \pm 0.19$ & \\
\hline $22649 \quad \ldots \ldots \ldots$ & BD Cam & 5.12 & $\mathrm{~S} 3$ & 65 & $\ldots \pm 0.37$ & SB1; symbiotic \\
\hline $25408 \ldots \ldots \ldots$ & UV Cam & 7.62 & $\mathrm{C} 5,3$ & 16 & $\ldots \pm 0.56$ & Self \\
\hline $25604 \ldots \ldots \ldots$ & 37 Tau & 4.36 & K0 III & 108 & $9.58 \pm 0.18$ & \\
\hline $26630 \ldots \ldots$ & $\mu$ Per & 4.14 & G0 Ib & 48 & $\ldots \pm 0.24$ & SB1 \\
\hline $26673 \ldots \ldots$. & 52 Per & 4.71 & $\mathrm{G} 5 \mathrm{Ib}+\mathrm{A} 2 \mathrm{~V}$ & 68 & $\ldots \pm 0.11$ & SB1 \\
\hline $29139 \ldots \ldots$. & $\alpha \mathrm{Tau}$ & 0.85 & K5 III & 121 & $54.48 \pm 0.22$ & \\
\hline $31398 \ldots \ldots \ldots$ & $\iota$ Aur & 2.69 & K3 II & 89 & $17.20 \pm 0.27$ & $P \sim 700$ days? \\
\hline $32068 \ldots \ldots \ldots$ & $\zeta$ Aur & 3.75 & $\mathrm{~K} 4 \mathrm{II}+\mathrm{B} 8 \mathrm{~V}$ & 242 & $\ldots \pm 0.24$ & SB1 \\
\hline $36167 \ldots \ldots \ldots$ & 31 Ori & 4.71 & K5 III & 123 & $6.90 \pm 0.17$ & \\
\hline $36389 \quad \ldots \ldots \ldots$ & 19 Tau & 4.38 & M2 Iab-Ib & 128 & $22.36 \pm 1.31$ & Multi-P \\
\hline $39801 \ldots \ldots \ldots$ & $\alpha$ Ori & 0.50 & M1-2 Ia-Iab & 153 & $21.55 \pm 1.87$ & \\
\hline $39816 \ldots \ldots$. & U Ori & 6.70 & M6.5 IIIe & 99 & $\ldots \pm 1.96$ & Mira; self \\
\hline $41116 \ldots \ldots$. & $1 \mathrm{Gem}$ & 4.16 & G7 III & 359 & $24.36 \pm 2.43$ & $\mathrm{P}>150$ days \\
\hline $42995 \ldots \ldots \ldots$ & $\eta \mathrm{Gem}$ & 3.28 & M3 III & 118 & $14.27 \pm 5.66$ & Long-P?; SB? \\
\hline $43039 \quad \ldots \ldots \ldots$ & $\kappa$ Aur & 4.35 & G8.5 IIIb & 92 & $20.73 \pm 0.14$ & \\
\hline $44478 \ldots \ldots \ldots$ & $\mu \mathrm{Gem}$ & 2.88 & M3 IIIab & 133 & $53.70 \pm 0.41$ & Irr \\
\hline $44537 \ldots \ldots \ldots$ & $\psi^{1}$ Aur & 4.91 & K5-M0 I & 69 & $1.51 \pm 1.82$ & Long-P? \\
\hline $48329 \quad \ldots \ldots \ldots$ & $\epsilon \mathrm{Gem}$ & 2.98 & G8 Ib & 110 & $7.80 \pm 0.33$ & \\
\hline $52973 \ldots \ldots \ldots$ & $\zeta \mathrm{Gem}$ & 3.79 & F7-G3 Ib & 130 & $5.52 \pm 8.83$ & Cepheid \\
\hline $54716 \ldots \ldots$ & 63 Aur & 4.90 & K4 III-IIIa & 75 & $-27.73 \pm 0.20$ & \\
\hline $55751 \ldots \ldots$ & & 5.35 & K2 II & 143 & $36.86 \pm 0.44$ & \\
\hline $59643 \ldots \ldots$. & NQ Gem & 8.01 & $\mathrm{R} 9$ & 44 & $\ldots \pm 1.49$ & Symbiotic; self \\
\hline $60522 \ldots \ldots$. & $v \mathrm{Gem}$ & 4.06 & M0 III-IIIb & 117 & $-21.62 \pm 0.19$ & \\
\hline $62044 \ldots \ldots \ldots$ & $\sigma \mathrm{Gem}$ & 4.28 & K1 III & 311 & $\ldots \pm 0.44$ & SB1; RS CVn \\
\hline $62345 \ldots \ldots \ldots$ & $\kappa \mathrm{Gem}$ & 3.57 & G8 IIIa & 153 & $20.35 \pm 0.33$ & Long-P? \\
\hline $62509 \quad \ldots \ldots \ldots$ & $\beta \mathrm{Gem}$ & 1.14 & K0 IIIb & 150 & $3.39 \pm 0.11$ & \\
\hline $69267 \ldots \ldots$. & $\beta \mathrm{Cnc}$ & 3.52 & K4 III & 192 & $22.70 \pm 0.21$ & \\
\hline $74442 \ldots \ldots \ldots$ & $\delta \mathrm{Cnc}$ & 3.94 & K0 III-IIIb & 124 & $16.93 \pm 0.15$ & \\
\hline $76294 \ldots \ldots \ldots$ & $\zeta$ Hya & 3.11 & G9 II-III & 139 & $22.78 \pm 0.14$ & \\
\hline $78712 \ldots \ldots$ & RS Cnc & 5.95 & M6 IIIase & 100 & $\ldots \pm 0.81$ & SR; self \\
\hline $80493 \ldots \ldots$ & $\alpha$ Lyn & 3.13 & K7 IIIab & 139 & $37.63 \pm 0.21$ & \\
\hline $81797 \ldots \ldots$ & $\alpha$ Hya & 1.98 & K3 II-III & 149 & $-4.21 \pm 0.19$ & \\
\hline $84441 \ldots \ldots$. & $\epsilon$ Leo & 2.98 & G1 II & 141 & $4.42 \pm 0.14$ & \\
\hline $89758 \quad \ldots \ldots \ldots$ & $\mu \mathrm{UMa}$ & 3.05 & M0 III & 118 & $\ldots \pm 0.38$ & SB1 \\
\hline $93813 \ldots \ldots$. & $\nu$ Нуа & 3.11 & K2 III & 144 & $-0.47 \pm 0.15$ & Long-P? \\
\hline $96833 \ldots \ldots$. & $\psi \mathrm{UMa}$ & 3.01 & K1 III & 97 & $-3.86 \pm 0.15$ & \\
\hline $102212 \ldots \ldots$ & $\nu$ Vir & 4.03 & M1 IIIab & 143 & $50.39 \pm 0.30$ & Long-P? \\
\hline $112300 \ldots \ldots$ & $\delta \mathrm{Vir}$ & 3.38 & M3 III & 139 & $-18.95 \pm 0.40$ & \\
\hline $112769 \ldots \ldots$ & 36 Com & 4.78 & M1 IIIb & 132 & $-1.65 \pm 0.29$ & \\
\hline $113226 \ldots \ldots$ & $\epsilon$ Vir & 2.83 & G8 IIIab & 146 & $-14.38 \pm 0.13$ & \\
\hline $115521 \ldots \ldots$ & $\sigma$ Vir & 4.80 & M2 IIIa & 135 & $-27.15 \pm 0.63$ & $P \sim 500$ days \\
\hline $122563 \ldots \ldots$ & & 6.20 & F8 IV & 30 & $-26.61 \pm 0.42$ & \\
\hline
\end{tabular}


TABLE 3 (Continued)

\begin{tabular}{|c|c|c|c|c|c|c|}
\hline $\begin{array}{l}\mathrm{HD} \\
(1)\end{array}$ & $\begin{array}{l}\text { Name } \\
(2)\end{array}$ & $\begin{array}{c}V \\
(3)\end{array}$ & $\begin{array}{c}\text { Spectral Type } \\
\text { (4) }\end{array}$ & $\begin{array}{l}N \\
(5)\end{array}$ & $\begin{array}{c}\mathrm{RV}_{\mathrm{AST}}^{\mathrm{a}} \\
\left(\mathrm{km} \mathrm{s}^{-1}\right) \\
(6)\end{array}$ & $\begin{array}{l}\text { Notes } \\
(7)\end{array}$ \\
\hline 124897 & $\alpha$ Boo & 0.04 & K1 III & 168 & $-5.20 \pm 0.21$ & $\mathrm{P} \sim 230$ days \\
\hline $127665 \ldots \ldots$ & $\rho$ Boo & 3.58 & K3 III & 111 & $-13.73 \pm 0.20$ & \\
\hline $129989 \ldots \ldots$ & $\epsilon$ Boo & 2.70 & K0 II-III & 132 & $-16.39 \pm 0.14$ & \\
\hline $133208 \ldots \ldots$ & $\beta$ Boo & 3.50 & G8 IIIa & 132 & $-16.39 \pm 0.14$ & \\
\hline $145931 \ldots \ldots$ & & 5.87 & $\mathrm{~K} 4 \mathrm{II}+\mathrm{F} 6-8 \mathrm{~V}$ & 59 & $-21.72 \pm 0.21$ & \\
\hline $146051 \ldots \ldots$ & $\delta \mathrm{Oph}$ & 2.74 & M0.5 III & 142 & $-19.46 \pm 0.24$ & Long-P? \\
\hline $148783 \ldots \ldots$ & $30 \mathrm{~g} \mathrm{Her}$ & 5.04 & M6 III & 57 & $0.23 \pm 1.30$ & \\
\hline $148856 \ldots \ldots$ & $\beta$ Her & 2.77 & G7 IIIa & 127 & $\ldots \pm 0.18$ & SB1 \\
\hline $156014 \ldots \ldots$ & $\alpha^{1}$ Her & 3.48 & M5 Ib-II & 136 & $-34.95 \pm 1.71$ & \\
\hline $156283 \ldots \ldots$ & $\pi$ Her & 3.16 & K3 IIab & 83 & $-25.59 \pm 0.23$ & \\
\hline $157999 \ldots \ldots$ & $\sigma \mathrm{Oph}$ & 4.34 & K2 II & 142 & $-27.39 \pm 0.24$ & \\
\hline $159181 \ldots \ldots$ & $\beta$ Dra & 2.79 & G2 Ib-IIa & 30 & $-20.90 \pm 0.28$ & \\
\hline $163770 \ldots \ldots$ & $\theta$ Her & 3.86 & K1 IIa & 66 & $-28.13 \pm 0.24$ & \\
\hline $164058 \ldots \ldots$ & $\gamma$ Dra & 2.23 & K5 III & 59 & $-27.65 \pm 0.17$ & \\
\hline $165195 \ldots \ldots$ & V2564 Oph & 7.34 & $\mathrm{~K} 3 \mathrm{p}$ & 27 & $-0.34 \pm 0.56$ & Long-P?; SB1 \\
\hline $168723 \ldots \ldots$ & $\eta$ Ser & 3.26 & K2 IIIab & 142 & $9.40 \pm 0.21$ & \\
\hline $173764 \ldots \ldots$ & $\beta$ Sct & 4.22 & G4 II+B9.5 & 118 & $\ldots \pm 0.23$ & SB1 \\
\hline $175865 \ldots \ldots$ & 13 Lyr & 4.13 & M5 III & 14 & $-29.00 \pm 1.11$ & Long-P? \\
\hline $180809 \ldots \ldots$ & $\theta$ Lyr & 4.36 & K0 II & 38 & $-31.48 \pm 0.16$ & \\
\hline $182040 \ldots \ldots$ & & 7.00 & $\mathrm{C} 1,2$ & 33 & $\ldots \pm 0.79$ & Self \\
\hline $183439 \ldots \ldots$ & $\alpha \mathrm{Vul}$ & 4.44 & M0 III & 97 & $-85.53 \pm 0.37$ & \\
\hline $183912 \ldots \ldots$ & $\beta^{1}$ Cyg & 3.08 & $\mathrm{~K} 3 \mathrm{II}+\mathrm{B} 0.5 \mathrm{~V}$ & 110 & $-24.99 \pm 0.22$ & \\
\hline $186791 \ldots \ldots$ & $\gamma \mathrm{Aql}$ & 2.72 & K3 II & 128 & $-2.68 \pm 0.45$ & Long-P? \\
\hline $187076 \ldots \ldots$ & $\delta$ Sge & 3.82 & $\mathrm{M} 2 \mathrm{II}+\mathrm{A} 0 \mathrm{~V}$ & 117 & $\ldots \pm 0.57$ & SB1 \\
\hline $189319 \ldots \ldots$ & $\gamma$ Sge & 3.47 & M0 III & 105 & $-33.73 \pm 0.26$ & \\
\hline $192577 \ldots \ldots$ & 31 Cyg & 3.79 & K2 II+B3 V & 191 & $\ldots \pm 0.26$ & SB1 \\
\hline $192713 \ldots \ldots$ & $22 \mathrm{Vul}$ & 5.15 & G3 Ib-II & 102 & Var. \pm 0.27 & SB1 \\
\hline $192876 \ldots \ldots$ & $\alpha^{1}$ Cap & 4.24 & G3 Ib & 128 & $-26.63 \pm 0.22$ & \\
\hline $192909 \ldots \ldots$ & 32 Cyg & 3.98 & K3 Ib+B3 V & 292 & $\ldots \pm 0.29$ & SB1 \\
\hline $194317 \ldots \ldots$ & 39 Cyg & 4.43 & K3 III & 65 & $-17.19 \pm 0.34$ & Long-P? \\
\hline $196093 \ldots \ldots$ & 47 Cyg & 4.61 & $\mathrm{~K} 2 \mathrm{Ib}+\mathrm{B} 3 \mathrm{~V}$ & 68 & $\ldots \pm 0.28$ & SB1 \\
\hline $196321 \ldots \ldots$ & $70 \mathrm{Aql}$ & 4.89 & K5 II & 116 & $-9.34 \pm 0.39$ & \\
\hline $197989 \ldots \ldots$ & $\epsilon$ Cyg & 2.46 & K0 III & 73 & $-12.87 \pm 0.17$ & Long-P? \\
\hline $200905 \ldots \ldots$ & $\xi \mathrm{Cyg}$ & 3.72 & K4-5 Ib-II & 66 & $-17.48 \pm 0.38$ & Long-P? \\
\hline $201251 \ldots \ldots$ & 63 Cyg & 4.55 & K4 Ib-IIa & 41 & $-26.55 \pm 0.19$ & \\
\hline $201626 \ldots \ldots$ & & 8.13 & $\mathrm{R} 5-\mathrm{CH}$ & 16 & $-150.82 \pm 0.73$ & $\mathrm{CH}$ star \\
\hline $202109 \ldots \ldots$ & $\zeta$ Cyg & 3.20 & G8 III-IIIa & 74 & $19.55 \pm 0.25$ & \\
\hline $204724 \ldots \ldots$ & $2 \mathrm{Peg}$ & 4.57 & M1 III & 92 & $-19.02 \pm 0.34$ & \\
\hline $204867 \ldots \ldots$ & $\beta$ Aqr & 2.91 & G0 Ib & 128 & $6.33 \pm 0.23$ & \\
\hline $206778 \ldots \ldots$ & $\epsilon \mathrm{Peg}$ & 2.39 & $\mathrm{~K} 2 \mathrm{Ib}$ & 122 & $3.85 \pm 0.67$ & Long-P?; SB? \\
\hline $206859 \ldots \ldots$ & 9 Peg & 4.34 & G5 Ib & 98 & $-22.97 \pm 0.22$ & \\
\hline $209750 \ldots \ldots$ & $\alpha \mathrm{Aqr}$ & 2.96 & G2 Ib & 128 & $6.86 \pm 0.24$ & \\
\hline $210745 \ldots \ldots$ & $\zeta$ Cep & 3.35 & $\mathrm{~K} 1.5 \mathrm{Ib}$ & 34 & $-18.05 \pm 0.34$ & \\
\hline $211388 \ldots \ldots$ & $1 \mathrm{Lac}$ & 4.13 & K3 II-III & 66 & $-8.50 \pm 0.22$ & \\
\hline $213310 \ldots \ldots$ & $5 \mathrm{Lac}$ & 4.36 & $\mathrm{M} 0 \mathrm{II}+\mathrm{B} 8 \mathrm{~V}$ & 54 & $-11.73 \pm 0.38$ & Long-P? \\
\hline $215182 \ldots \ldots$ & $\eta$ Peg & 2.94 & G2 II-III+F & 75 & $\ldots \pm 0.14$ & SB1 \\
\hline $216131 \ldots \ldots$ & $\mu$ Peg & 3.48 & G8 III & 85 & $13.77 \pm 0.12$ & \\
\hline $216489 \ldots \ldots$ & IM Peg & 5.64 & K1 III & 322 & $\ldots \pm 0.34$ & SB1 \\
\hline $216946 \ldots \ldots$ & V424 Lac & 4.95 & K5 Ib & 65 & $-9.45 \pm 0.47$ & Long-P?; SB? \\
\hline $217906 \ldots \ldots$ & $\beta \mathrm{Peg}$ & 2.42 & M2.5 II-III & 95 & $8.39 \pm 0.95$ & SB? \\
\hline $219615 \ldots \ldots$ & $\gamma$ Psc & 3.69 & K0 III & 104 & $-14.34 \pm 0.13$ & \\
\hline $221170 \ldots \ldots$ & & 7.71 & G2 IV & 19 & $-121.63 \pm 0.16$ & \\
\hline $222107 \ldots \ldots$ & $\lambda$ And & 3.82 & G8 III & 140 & $\ldots \pm 0.26$ & RS CVn \\
\hline $223075 \ldots \ldots$ & TX Psc & 5.04 & C II... & 54 & $\ldots \pm 2.16$ & Self \\
\hline $224014 \ldots \ldots$ & $\rho$ Cas & 4.54 & G2 $0 \mathrm{e}$ & 52 & $-62.85 \pm 5.55$ & Long-P? \\
\hline $224935 \ldots \ldots$ & $30 \mathrm{Psc}$ & 4.41 & M3 III & 103 & $-12.05 \pm 0.43$ & \\
\hline $225212 \ldots \ldots$ & $3 \mathrm{Cet}$ & 4.94 & K3 Ibv & 97 & $-41.95 \pm 0.37$ & \\
\hline
\end{tabular}

NotEs. $-\mathrm{SB} 1$ = single-lined spectroscopic binary; SB? = suspected spectroscopic binary; Self $=$ velocity with respect to a spectrum of the star itself; RS CVn = RS CVn binary; Long-P? = stars with long-term variations greater than their short-term fluctuations; others should be obvious. For the binaries listed in Table 4, col. (6) gives the standard deviation with respect to the orbital solution.

${ }^{\text {a }}$ Velocity on IAU system. 


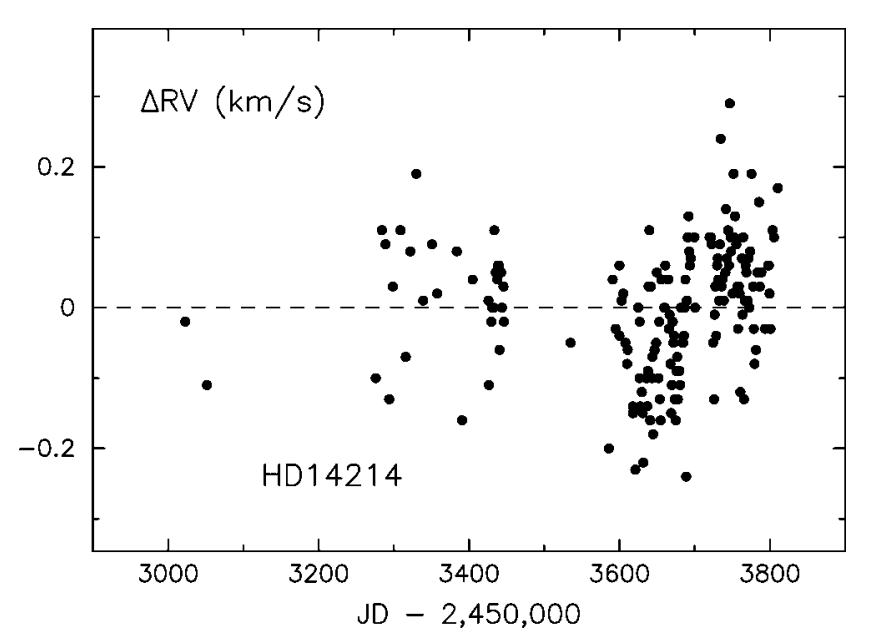

FIG. 5.-Deviations from a fi tted velocity curve for HD 14214. The standard deviation for these data is $0.10 \mathrm{~km} \mathrm{~s}^{-1}$.

Almost all the evolved G, K, and M stars we have observed show velocity variations at our level of precision. To assess just how ubiquitous this variability is, we must defi ne criteria for judging whether a star might be constant. Obviously, we are not going to detect any variability less than our likely external error of measurement $\left(\sigma_{\mathrm{ext}} \approx 0.11 \mathrm{~km} \mathrm{~s}^{-1}\right)$; stars with this level of variation are effectively constant. Let us establish a criterion for judging whether a star's observed variation is intrinsic or may be instrumental. This means determining just
TABLE 5

LEVELS OF VARIABILITY

\begin{tabular}{cccc}
\hline \hline Spectral Type & $\begin{array}{c}\text { Constant } \\
(\sigma=0.10-0.12)\end{array}$ & $\begin{array}{c}\text { Variable? } \\
(\sigma=0.13-0.14)\end{array}$ & $\begin{array}{c}\text { Variable } \\
(\sigma>0.14)\end{array}$ \\
\hline G0-G2 $\ldots \ldots \ldots \ldots$ & $\ldots$ & 2 & 7 \\
G3-G5 $\ldots \ldots \ldots \ldots$ & 1 & $\ldots$ & 3 \\
G6-G9 $\ldots \ldots \ldots \ldots$ & 1 & 5 & 5 \\
K0, K1 $\ldots \ldots \ldots$. & 1 & 2 & 10 \\
K2 $\ldots \ldots \ldots \ldots \ldots$ & 1 & $\ldots$ & 10 \\
K3 $\ldots \ldots \ldots \ldots \ldots$ & $\ldots$ & $\ldots$ & 14 \\
K4 $\ldots \ldots \ldots \ldots \ldots$ & $\ldots$ & $\ldots$ & 6 \\
K5-K7 $\ldots \ldots \ldots$. & $\ldots$ & $\ldots$ & 10 \\
M0-M1 $\ldots \ldots \ldots$. & $\ldots$ & $\ldots$ & 11 \\
M2-M3 $\ldots \ldots \ldots$. & $\ldots$ & $\ldots$ & 9 \\
M4-M5 $\ldots \ldots \ldots$. & $\ldots$ & $\ldots$ & 3 \\
$>$ M5, C, S $\ldots \ldots$. & $\ldots$ & 9 & 10 \\
All $\ldots \ldots \ldots \ldots$. & 4 & & 98
\end{tabular}

how much more than $\sigma_{\text {ext }}$ the standard deviation per observation, $\sigma_{\text {obs }}$, must be for the star to be considered variable. This $\sigma_{\text {obs }}$ is itself a measured quantity, which should become increasingly accurate with more observations, so it ought to be known to $1 / \sqrt{ } N$, or $\sigma_{\sigma} \approx 0.11 / \sqrt{ } 104=0.011 \mathrm{~km} \mathrm{~s}^{-1}$ for a typical star in Table 3. We thus propose to classify the variability by the following criteria: (1) stars that are effectively constant, $\sigma=\sigma_{\text {ext }}+\sigma_{\sigma}=0.10-0.12 \mathrm{~km} \mathrm{~s}^{-1}$; (2) stars that may be variable and deserve further consideration, $\sigma \leq \sigma_{\text {ext }}+$ $3 \sigma_{\sigma}$, or $0.13-0.14 \mathrm{~km} \mathrm{~s}^{-1}$; and (3) stars that are clearly variable, $\sigma>\sigma_{\text {ext }}+3 \sigma_{\sigma}=0.14 \mathrm{~km} \mathrm{~s}^{-1}$. Applying these criteria to our data set, we get the distribution in Table 5. This mimics the

TABLE 4

New Orbits for Spectroscopic Binaries

\begin{tabular}{|c|c|c|c|c|c|c|c|c|c|c|c|}
\hline HD & Name & $N$ & $\begin{array}{l}\text { Period } \\
\text { (days) }\end{array}$ & $\begin{aligned} & T^{\mathrm{a}} \\
\mathrm{HJD}- & 2,400,000\end{aligned}$ & $\begin{array}{c}K \\
\left(\mathrm{~km} \mathrm{~s}^{-1}\right)\end{array}$ & $\begin{array}{c}\gamma^{\mathrm{b}} \\
\left(\mathrm{km} \mathrm{s}^{-1}\right)\end{array}$ & & $e$ & $\begin{array}{c}\omega \\
(\mathrm{deg})\end{array}$ & $\begin{array}{c}\sigma^{\mathrm{c}} \\
\left(\mathrm{km} \mathrm{s}^{-1}\right)\end{array}$ & Refs. \\
\hline 352 & $5 \mathrm{Cet}$ & 126 & $(96.41)$ & $52,950.969 \pm 0.029$ & $23.94 \pm 0.05$ & $-0.73 \pm 0.04$ & 0.0328 & \pm 0.002 & $88.3 \pm 0.12$ & 0.39 & 1 \\
\hline 4502 & $\zeta$ And & 114 & $(17.7692)$ & $53,600.028 \pm 0.098$ & $25.95 \pm 0.08$ & $-24.53 \pm 0.04$ & & $\ldots$ & $\ldots$ & 0.43 & \\
\hline 22649 & BD Cam & 65 & $(596.21)$ & $52,967.73 \pm 0.78$ & $8.48 \pm 0.10$ & $-23.39 \pm 0.05$ & 0.099 & \pm 0.008 & $347.0 \pm 4.6$ & 0.38 & \\
\hline 26630 & $\mu$ Per & 48 & $(283.272)$ & $53,192.31 \pm 0.13$ & $19.62 \pm 0.07$ & $8.54 \pm 0.04$ & 0.055 & \pm 0.003 & $278.6 \pm 2.6$ & 0.25 & \\
\hline $26673 \ldots \ldots$ & 52 Per & 68 & (1576.44) & $52,742.00 \pm 0.57$ & $(18.10)$ & $-5.05 \pm 0.03$ & 0.364 & \pm 0.004 & $71.4 \pm 0.2$ & 0.12 & \\
\hline 32068 & $\zeta$ Aur & 242 & (972.162) & $53,039.578 \pm 0.11$ & $23.10 \pm 0.03$ & $10.77 \pm 0.02$ & 0.3982 & \pm 0.0009 & $328.9 \pm 0.14$ & 0.24 & 2 \\
\hline 62044 & $\sigma \mathrm{Gem}$ & 311 & (19.60447) & $47,232.052 \pm 0.003$ & $34.58 \pm 0.04$ & $42.91 \pm 0.02$ & & $\ldots$ & $\ldots$ & 0.44 & \\
\hline 89758 & $\mu \mathrm{UMa}$ & 118 & $(230.089)$ & $53,180.27 \pm 0.30$ & $7.69 \pm 0.06$ & $-21.48 \pm 0.04$ & & $\ldots$ & $\ldots$ & 0.40 & \\
\hline $148856 \ldots$ & $\beta$ Her & 127 & $(410.575)$ & $53,315.58 \pm 0.12$ & $13.11 \pm 0.03$ & $-26.13 \pm 0.02$ & 0.561 & \pm 0.001 & $23.0 \pm 0.3$ & 0.18 & \\
\hline 173764 & $\beta$ Sct & 118 & $(834)$ & $53,310.49 \pm 0.28$ & $14.76 \pm 0.04$ & $-22.06 \pm 0.03$ & 0.326 & \pm 0.002 & $35.0 \pm 0.5$ & 0.24 & \\
\hline $187076 \ldots \ldots$ & $\delta$ Sge & 117 & $(3700.00)$ & $(51,705.0)$ & $(8.02)$ & $0.53 \pm 0.05$ & $(0.44)$ & & $(257.7)$ & 0.57 & 3 \\
\hline 192577 & 31 Cyg & 191 & $(3784.34)$ & $(52,325.7)$ & (13.98) & $-7.65 \pm 0.04$ & $(0.228)$ & & (201.4) & 0.27 & 2 \\
\hline 192713 & $22 \mathrm{Vul}$ & 99 & $(249.131)$ & $53,229.26 \pm 0.05$ & $27.38 \pm 0.04$ & $-20.50 \pm 0.02$ & & . & $\cdots$ & 0.27 & 4 \\
\hline 192909 & $32 \mathrm{Cyg}$ & 292 & $(1147.80)$ & $33,139.48 \pm 0.26$ & $16.77 \pm 0.03$ & $-7.35 \pm 0.02$ & 0.3169 & \pm 0.0014 & $223.6 \pm 0.3$ & 0.29 & 2 \\
\hline 196093 & 47 Cyg & 68 & $(1117)$ & $53,659.4 \pm 4.2$ & $(2.00)$ & $-4.50 \pm 0.01$ & & $\ldots$ & $\ldots$ & 0.30 & 5 \\
\hline $215182 \ldots \ldots$ & $\eta \mathrm{Peg}$ & 75 & $(818.0)$ & $52,865.23 \pm 0.25$ & $14.39 \pm 0.02$ & $3.85 \pm 0.04$ & 0.171 & \pm 0.002 & $355.4 \pm 0.6$ & 0.14 & \\
\hline $216489 \ldots \ldots$ & IM Peg & 322 & $(24.64877)$ & $52,241.970 \pm 0.004$ & $34.93 \pm 0.04$ & $-14.15 \pm 0.03$ & 0.0317 & \pm 0.001 & $106.4 \pm 2.2$ & 0.38 & 6 \\
\hline $222107 \ldots \ldots$ & $\lambda$ And & 140 & (20.5212) & $53,601.010 \pm 0.015$ & $6.58 \pm 0.03$ & $6.44 \pm 0.02$ & 0.063 & \pm 0.005 & $321.2 \pm 4.2$ & 0.26 & 7 \\
\hline
\end{tabular}

Notes. - Values in parentheses are assumed values taken from the literature. Periods are generally from Batten et al. (1989) or Strassmeier et al. (1993).

References. -(1) Lines et al. 1984; (2) J. A. Eaton et al. 2007, in preparation; (3) Eaton et al. 1995; (4) Eaton \& Shaw 2007; (5) Griffi n 1992; (6) Marsden et al. 2005; (7) Walker 1944.

${ }^{a}$ Periastron (or ascending node for circular orbit).

${ }^{\mathrm{b}}$ Velocity on IAU system.

${ }^{c}$ Standard deviation of single datum from fi t. 
photometric result of Henry et al. (2000, Table 6) but is more extreme. Only $4 \%$ of the stars in Table 5 are constant at our level of precision, while roughly half the stars analyzed photometrically by Henry et al. could be constant by their criteria. Furthermore, two of the "constant" stars, $\alpha$ Ari and $\beta$ Gem, are known to be variable at a low level. At this point, we cannot say whether the $\mathrm{G}$ and $\mathrm{K}$ giants have organized radial pulsations detected in their variable velocities, because the periods expected are too short to have been effectively detected in our data. The median period for the radial fundamental for G7-K4 giants given by Henry et al. (2000, Table 7), for example, is near 4 days. A further check on the short-term variability is to look at the time variation and decide whether it looks random. Most of these stars show apparently coherent velocity variations of the order of a cycle or two long. According to G. W. Henry (2007, private communication), the plotted velocities look very much like the photometric variations of semiregular variables (Percy et al. 2001).

The cooler giants often have secondary periods much longer than their radial fundamental periods, which cannot be explained adequately by any known mechanism (Wood et al. 2004; Derekas et al. 2006). Such periods were seen convincingly in a large fraction $(\sim 25 \%)$ of stars sampled in large surveys of red variables, such as Wood et al. (1999). Our data do not cover a long enough time span ( $\$ 1000$ days) to see just how prevalent long-term variations are in the $\mathrm{K}$ stars, but we have identifi ed 19 stars in Table 3 with long-term velocity variation at least as large as their short-term fluctuations. A few of these may be spectroscopic binaries, as we have suggested in the notes to the table, but much more observing would be necessary to prove that.

\subsection{Spectroscopic Binaries}

The sample included a number of spectroscopic binaries, many with rather long periods, and we have fi t orbits to their velocity curves to get the elements in Table 4. In doing this, we assumed orbital periods from the literature, because our data did not cover enough time to defi ne the periods. In principle, we could have combined our new data with published data, generally of much lower statistical weight, to obtain improved periods, but that approach would have introduced arbitrary zero-point corrections and increased the uncertainties of the other orbital elements derived.

Most of the eccentricities are probably real, but two of them are likely spurious. The single-lined tidally distorted binary 5 Cet (= HD 352) has a decidedly nonsinusoidal velocity curve that repeats over several cycles. Our attempts to fi $t$ it with a circular orbit gave $\sigma=0.69 \mathrm{~km} \mathrm{~s}^{-1}$. We could not even fi t it to within the expected errors with an elliptical orbit $(\sigma=$ 0.39). Much of the elevated error in both these attempts is caused by a wave in the residuals with the right phase dependence and amplitude for the signature of tidal distortion (Sterne 1941); scatter about this wave is close to the $0.10 \mathrm{~km} \mathrm{~s}^{-1}$ external error of the spectrograph (see Eaton 2007). The eccentricity in IM Peg, on the other hand, is more likely caused by distortions of its lines by starspots, an effect for which there can be no theory.

\section{SUMMARY}

We have discussed flat-fi elding and wavelength calibration for spectroscopy in the context of data reduction for the Tennessee State automatic telescope, suggesting that obtaining fht-fi eld images with the spectrograph out of focus is a good way of more uniformly illuminating the CCD and getting a better determined measurement of pixel-to-pixel variation in sensitivity.

We have discussed the IAU velocity system as an analogy to the $U B V$ photometric system, argued for applying it in an intellectually honest way to establish zero points for particular spectrographs, and would argue further that this approach is necessary to knowing the relative shifts of various observers' velocities well enough to piece them together reliably into a single velocity curve. We have presented a consistent, automated technique for measuring radial velocities for cool stars earlier than middle $\mathrm{M}$ spectral type. The transformation of these velocities to the IAU radial velocity system is +0.35 $\pm 0.09 \mathrm{~km} \mathrm{~s}^{-1}$.

We have established the external error for the TSU spectrograph by using many data for the most constant cool stars we have observed, fi nding a standard deviation per observation that tends asymptotically to $\sim 0.10 \mathrm{~km} \mathrm{~s}^{-1}$ with decreasing stellar variability. We are formally quoting a value of $\sigma_{\text {ext }}=$ $0.10-0.11 \mathrm{~km} \mathrm{~s}^{-1}$ for the spectrograph. This value seems to apply for both sharp- and broad-lined stars, with the greater variability of velocities of the broad-lined stars caused by intrinsic variation of those stars.

We have presented results of monitoring about $120 \mathrm{cool}$ stars over the fi rst 2-3 years of a so-called synoptic program. Variation of our extensive radial velocities agrees with the notion that all the cool giants $(\mathrm{G}-\mathrm{M})$ are variable. The $\mathrm{G} 6-\mathrm{K} 2$ giants, which seem to be in a region of minimal photometric variability in the HR diagram, are $90 \%$ variable or possibly variable in our radial velocities, for example. Such stars seem variable from a variety of mechanisms, mostly understood poorly at best (e.g., Wood et al. 2004; Brown 2007), and we expect that variability will continue being an exciting topic of research in the future.

We have also given improved elements for 18 long-period spectroscopic binaries.

We would like to thank Jeff Hall for the work he did in adapting his data reduction program to our application, and Harland Epps for graciously helping us fi nish the TSU spectrograph. We also thank Colin Scarfe for helpfully discussing the IAU velocity system with us, and F. C. Fekel for providing some useful references to work on standardization. This re- 
search used the SIMBAD database extensively and was sup-

ported by NASA grants NCCW-0085 and NCC5-511 and by NSF grants HRD 9550561, HRD 9706268, and AST 0618404.

\section{REFERENCES}

Balthasar, H., Thiede, U., \& Wohl, H. 1982, A\&A, 114, 357

Batten, A. H., Fletcher, J. M., \& MacCarthy, D. G. 1989, Publ. Dominion Astrophys. Obs. Victoria, 17, 1

Batten, A. H., Harris, H. C., McClure, R. D., \& Scarfe, C. D. 1983, Publ. Dominion Astrophys. Obs. Victoria, 16, 143

Bizyaev, D., \& Smith, V. V. 2007, PASP, 119, 143

Bouigue, R. 1973, Trans. IAU, 15A, 407

Boyle, R. P., Janusz, R., Laugalys, V., \& Philip, A. G. D. 2003, BAAS, 35, 1207

Brown, K. I. T. 2007, PASP, 119, 237

Cummings, I. N., Hearnshaw, J. B., Kilmartin, P. M., \& Gilmore, A. C. 1999, in ASP Conf. Ser. 185, Precise Stellar Radial Velocities, ed. J. B. Hearnshaw \& C. D. Scarfe (San Francisco: ASP), 204

Dekker, H., Delabre, B., Hess, G., \& Kotzlowski, H. 1992, in Progress in Telescope and Instrumentation Technologies, ed. M.-H. Ulrich (Garching: ESO), 581

Deming, D., Espenak, F., Jennings, D. E., Brault, J. W., \& Wagner, J. 1987, ApJ, 316, 771

Derekas, A., Kiss, L. L., Bedding, T. R., Kjeldsen, H., Lah, P., \& Szabo, Gy. M. 2006, ApJ, 650, L55

Eaton, J. A. 2007, ApJ, submitted

Eaton, J. A., Hartkopf, W. I., McAlister, H. A., \& Mason, B. D. 1995, AJ, 109, 1856

Eaton, J. A., Henry, G. W., \& Fekel, F. C. 2003, in The Future of Small Telescopes in the New Millennium, Vol. II, The Telescopes We Use, ed. T. D. Oswalt (Dordrecht: Kluwer), 189

Eaton, J. A., \& Shaw, F. G. 2007, AJ, 133, 2669

Eaton, J. A., \& Williamson, M. H. 2004a, Proc. SPIE, 5496, 710 2004b, Astron. Nachr., 325, 522

Epps, H. W. 1998, Proc. SPIE, 3555, 111

Fekel, F. C., Williamson, M., \& Pourbaix, D. 2007, AJ, 133, 2431

Gray, D. F., \& Brown, K. I. T. 2006, PASP, 118, 399

Gray, D. F., \& Toner, C. G. 1985, PASP, 97, 543

1986a, PASP, 98, 499

1986b, ApJ, 310, 277

Griffi n, R. F. 1992, Observatory, 112, 111

Griffi n, R., \& Griffi n, R. 1973, MNRAS, 162, 243

Hall, D. S. 1976, in IAU Colloq. 29, Multiple Periodic Variable Stars, Part I, ed. W. S. Fitch (Dordrecht: Reidel), 287

Hall, J. C., \& Lockwood, G. W. 1995, ApJ, 438, 404

Hall, J. C., Fulton, E. E., Huenemoerder, D. P., Welty, A. D., \& Neff, J. E. 1994, PASP, 106, 315

Hatzes, A. P, \& Cochran, W. D. 1993, ApJ, 413, 339
Henry, G. W. 1995, in ASP Conf. Ser. 79, Robotic Telescopes: Current Capabilities, Present Developments, and Future Prospects for Automated Astronomy, ed. G. W. Henry \& J. A. Eaton (Provo: ASP), 44

Henry, G. W., Fekel, F. C., Henry, S. M., \& Hall, D. S. 2000, ApJS, 130, 201

Johnson, H. L., \& Morgan, W. W. 1953, ApJ, 117, 313

Kamper, K. W. 1996, JRASC, 90, 140

Kamper, K. W., \& Fernie, J. D. 1998, AJ, 116, 936

Kobanov, N. I. 1985, Sol. Phys., 99, 21

Larson, A. M., Yang, S. L. S., \& Walker, G. A. H. 1999, in ASP Conf. Ser. 185, Precise Stellar Radial Velocities, ed. J. B. Hearnshaw \& C. D. Scarfe (San Francisco: ASP), 193

Libbrecht, K. G., \& Peri, M. L. 1995, PASP, 107, 62

Lines, R. D., Boyd, L. J., Genet, R. M., \& Hall, D. S. 1984, Inf. Bull. Variable Stars, 2589

Marsden, S. C., et al. 2005, ApJ, 634, L173

Moore, C. E., Minnaert, M. G. J., \& Houtgast, J. 1966, The Solar Spectrum $2935 \AA$ to $8770 \AA$ (NBS Monogr. 61; Washington: USGPO)

Pearce, J. A. 1957, Trans. IAU, 9, 441

Percy, J. R., Wilson, J. B., \& Henry, G. W, 2001, PASP, 113, 983

Pilachowski, C., Dekker, H., Hinkle, K., Tull, R., Vogt, S., Walker, D. D., Diego, F., \& Angel, R. 1995, PASP, 107, 983

Queloz, D., et al. 2001, A\&A, 379, 279

Scarfe, C. D. 1985, in IAU Symp. 111, Calibration of Fundamental Stellar Quantities, ed. D. S. Hayes, L. E. Pasinetti, \& A. G. Davis Philipi (Dordrecht: Reidel), 583

Scarfe, C. D., Batten, A. H., \& Fletcher, J. M. 1990, Publ. Dominion Astrophys. Obs. Victoria, 18, 21

Smith, M. A., Patten, B. M., \& Goldberg, L. 1989, AJ, 98, 2233

Stefanek, R. P., Latham, D. W., \& Torres, G. 1999, in ASP Conf. Ser. 185, Precise Stellar Radial Velocities, ed. J. B. Hearnshaw \& C. D. Scarfe (San Francisco: ASP), 354

Sterne, T. C. 1941, Proc. Natl. Acad. Sci., 27, 168

Strassmeier, K. G., Hall, D. S., Fekel, F. C., \& Scheck, M. 1993, A\&AS, 100, 173

Walker, E. C. 1944, JRASC, 38, 249

Walker, G. A. H., Yang, S., Campbell, B., \& Irwin, A. W. 1989, ApJ, 343, L21

Wood, P. R., Olivier, E. A., \& Kawaler, S. D. 2004, ApJ, 604, 800

Wood, P. R., et al. 1999, in IAU Symp. 191, Asymptotic Giant Branch Stars, ed. T. Le Bertre, A. Lebre, \& C. Waelkens (San Francisco: ASP), 151 